\title{
Evaluating emergency preparedness with exercise monitoring
}

\author{
D. Marten, S. Weiss, M. Stiehl, K. Roth, O. A. Mudimu \\ \& A. Lechleuthner \\ Institute of Rescue Engineering and Civil Protection, \\ Cologne University of Applied Sciences, Germany
}

\begin{abstract}
This paper describes a methodology of exercise monitoring using qualitative and quantitative tools. These tools document the exercise time flow, gauge appropriate measures and decisions and collect feedback of exercise participants. These three elements provide a basis to assess the quality of the undertaken measures. The documentation of the operational performance helps to enhance emergence preparedness. This research has great potential to lead to operational insights at the micro-level.

Keywords: exercise monitoring, evaluating emergency exercise, TeleDialogsystem, local positioning, rescue team.
\end{abstract}

\section{Introduction}

Beginning with the preparations for the 2006 Football World Cup in Germany, there have been a lot of innovations in the sector of disaster preparedness in Germany [1]. Especially the scenario of a mass casualty incident during the World Cup was feared and therefore one of the main topics in the preparation of public authorities [2,3]. Thus many of the hosting cities overhauled their protocols and concepts for emergency medical services (EMS) and fire brigades or drafted completely new ones that took locally available resources and facilities into consideration. Regarding the FIFA guidelines, the amount of patients expected in mass casualty incidents was increased to 1000 and more casualties [4]. However, before implementing new concepts, both training and testing are required. Therefore many practical exercises were held to check or verify the efficiency of these new protocols $[4,5]$. In addition to these full 
practical exercises with real emergency personnel and patient performers, so called tabletop exercises focusing on emergency management and simulating the real treatment of patients were also held.

All kinds of exercises need a lot of preparation and post processing to be an efficient help to the implementing authorities. To verify the functionality of an operations schedule, independent and objective evaluation is needed [6]. Members of the Institute of Rescue Engineering and Civil Protection at the Cologne University of Applied Sciences started to evaluate exercises back in 2008 while working on a research project called MANET. The aim of MANET was to develop a system of autonomic cross-linked devices to support rescue teams coping with mass casualty incidents [7]. During the research project, it became necessary to measure and document the impact of developed devices within the process of rescue. Therefore it was decided to evaluate exercises in which the devices were tested. A standardized evaluation system was developed to gain and review comparable data.

Within MANET, the Institute of Rescue Engineering and Civil Protection developed a methodology of exercise monitoring including qualitative and quantitative evaluation. The main aim of this methodology is to compare the protocol and its functionality under real circumstances. Eventually the measurements and reports become the basis for drafting an evaluation report afterwards.

The developed methodology was implemented and tested within the project MANET and its follow-up project evalMANV. Both research projects have been funded by the German Federal Ministry for Education and Research The aim of evalMANV is to analyse different concepts and protocols for mass casualty incidents and compare their capabilities. Since emergency management in Germany is the responsibility of the local governments, the strategies and concepts that are used to cope with mass casualty incidents differ greatly depending on the guidelines of the sixteen federal states $[8,9]$. These differences complicate the process of analysing and comparing different concepts since the methodology needs to be adapted to both the scenario and the applied concept [10].

Within the following sections, we will give a more detailed view on the developed methodology itself with its quantitative and qualitative components. The functionality and implementation of external technologies will also be explained. Finally we will have a look at the results gained with the developed evaluation method and conclude with discussion and further plans for advancing the methodology.

\section{Methods}

The current evaluation method is based on three different elements

1. documenting the exercise time flow

2. gauging the appropriate measures and decisions

3. collecting feedback of exercise participants 
These three elements provide a basis to assess the quality of the undertaken measures.

Using the time flow documentation, several important actions during a mass casualty incident such as times of arrival for different ambulances or rescue teams, speed of triage process or time of rescue are recorded. This procedure provides an exercise log that allows us to recapitulate the whole exercise. It is possible to recap the course of the exercise after the event and thus to understand exactly what happened. This might be able to explain why certain things happen and can identify strengths or weaknesses of operations concept. As an example, a sub-process is graphically displayed below.

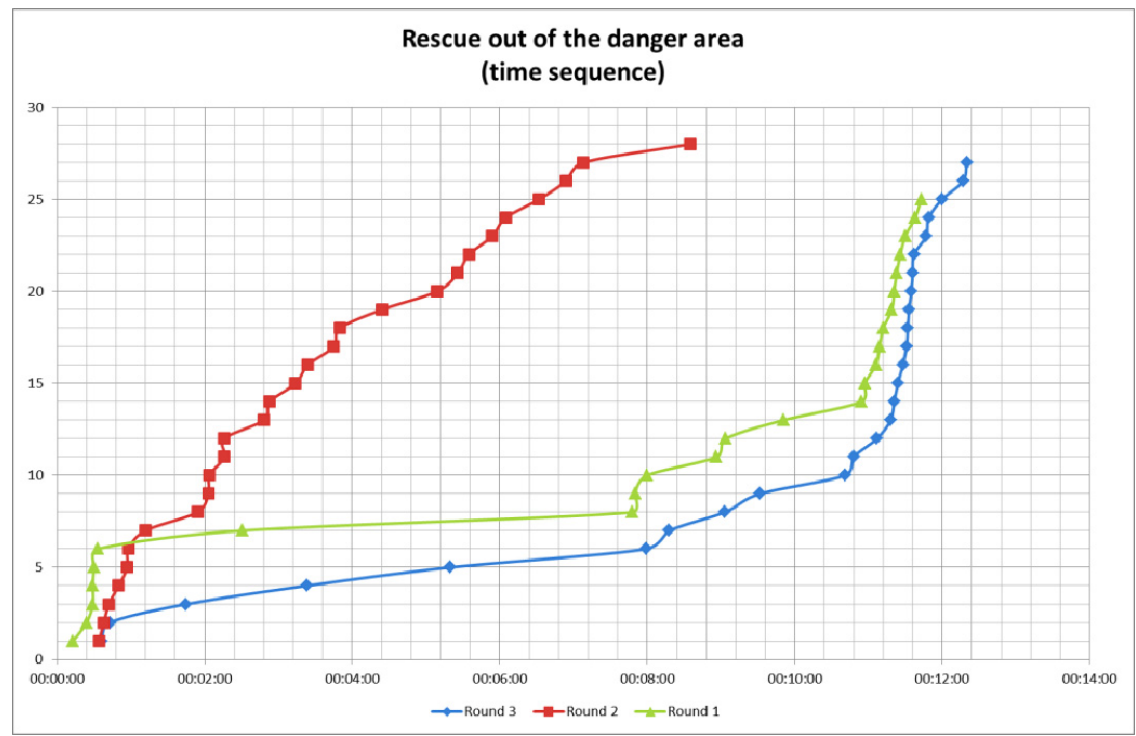

Figure 1: $\quad$ Rescue out of the danger area.

All in all, the sub-processes can represent and show how the overall process of the exercise is/was. In the data set summarized here, quantitative metrics, which can be collected manually and technically, are used exclusively. The survey is based on their technical accuracy and is recommended everywhere that it is feasible.

\subsection{Gauging appropriate measures and decisions}

In addition, the accuracy of implemented measures of the rescue squad is measured. This dimension is parallel to the speed an important dimension. The measures that are interesting are triage, rescue and provision of pre-hospital care. Top quality triage is a prerequisite for a successful mission [3]. 


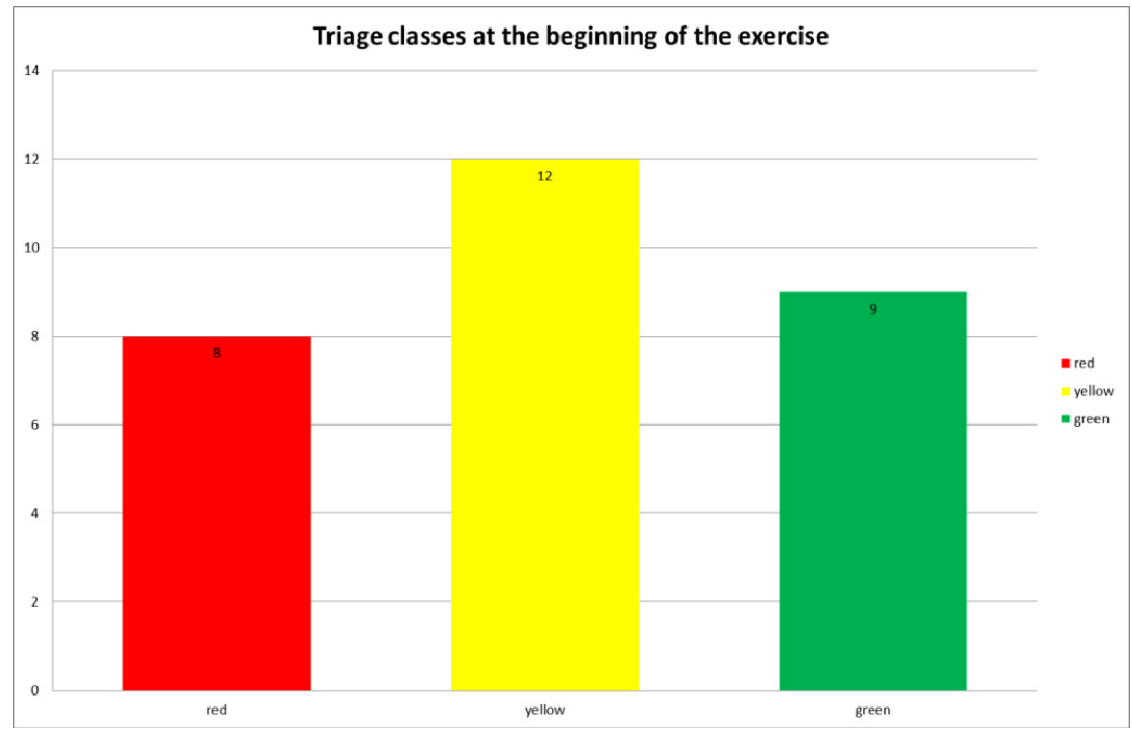

Figure 2: $\quad$ Triage classes at the beginning of the exercise.

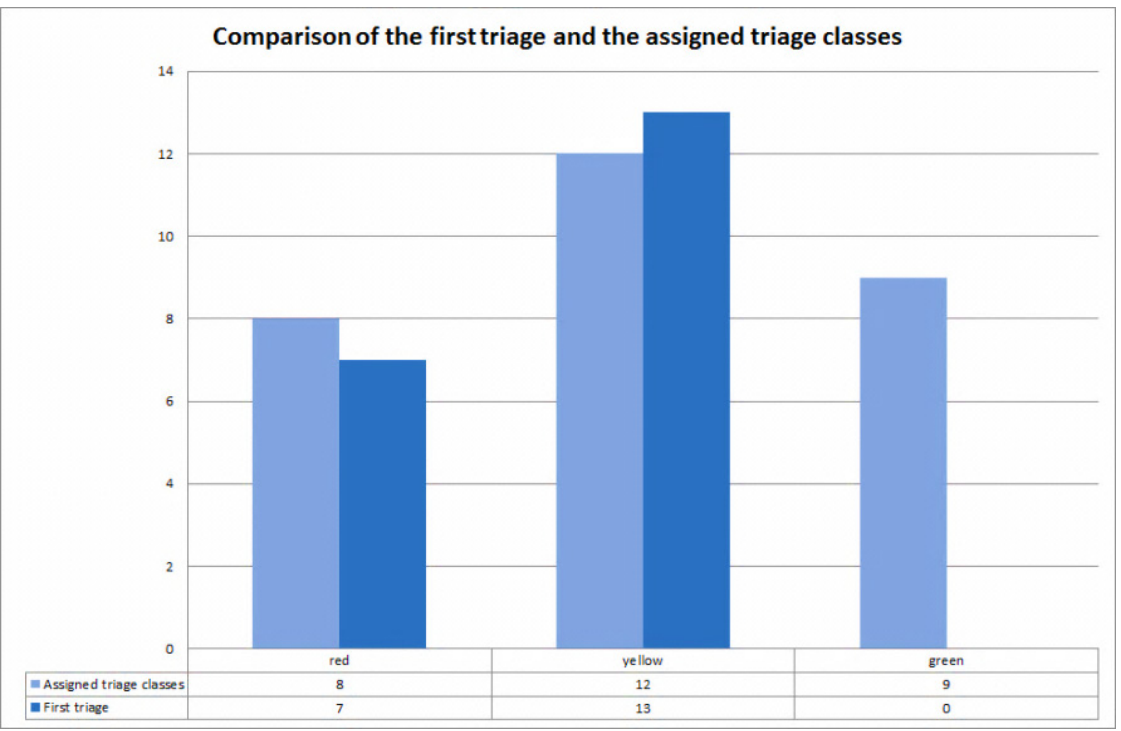

Figure 3: Comparison of the first triage and the assigned triage classes.

These data contain both quantitative and qualitative tools, and include feedback from exercise participants and patient performers.

Both tools are described in detail in the following section. 


\subsection{Quantitative tools}

The TeleDialog-System (TED-System) is a product, which has been used by the Cologne University of Applied Sciences in practicing large-scale disaster scenarios for about three years. It is a query tool, based on radio waves, which was originally used in the entertainment branch to process votes from large numbers of visitors. There, the application areas were mainly large events that needed answers to pre-programmed or imported questions and also to facilitate choices. By using an access point and omnidirectional antennas, a Wireless Local Area Network (WLAN) is built up, which is secured against external interventions by a dongle and its enclosed codification of the producer.

In the exercises evaluated by the Cologne University of Applied Sciences, the patient performers are briefly told what to do and equipped with the TED device before the scenario starts. During the exercise, they have to answer questions, which are displayed on the system with the multiple-choice method. To do this, the system offers several techniques: On the one hand, it is possible to program the questions with a different number of optional answers and the necessary analysis previously. Furthermore in a so-called live-mode, it is also possible to only install single questions on the gadgets. For the analysis, the transmission of data, which is split up into possible answers, time and number of identification, is of special importance. Through software technological settings and preliminary planning, it is possible to differentiate the data set concerning scenarios and processes separately.

Before using the system, the local setting in which the exercise takes place has to be considered. The system has to be planned and regulated with respect to the expected answers that are obtained from the TED questions. For regulating such situations, the Cologne University of Applied Sciences has developed a software solution that shows the recorded data of the singular exercises and the achieved observations in real time. The method with which the information is stored there in a data file has to be determined and defined by the responsible person. Due to this list of data, it is possible to make statements about various factors only seconds after the exercise, such as the point of time when the sick person was discovered or the number of people that took care of the same person. Thus, the practicing people can receive a positive or negative feedback, which can be useful in further runs.

By using a projector or different technologies, it is additionally possible to inform the observers about the duration of the exercise. Thus the organizing and analysing of the observers' reports is facilitated because all observers record the operational measures at the same time. Before each exercise, the accommodations and a contract document are needed for the evaluation, in order to plan the scenario exactly and program the technology.

\subsection{Qualitative tools}

Before the trial run, the observers were instructed in the exercise scenario. They received detailed information about the aim and the objectives of the exercise and the expectations regarding the participant rescue teams. 
To be able to assess all implemented operational measures, all observers had to fill in standardized form during or after the exercise. The observers must be deployed purposefully to notice all relevant processes of the operation.

The observation is divided into different steps. In the first step, observation, information is collected and processed cognitively. The behaviour of the rescue teams will be value-neutral, unbiased and watched without any emotional distortion. Written documentation is obtained immediately since the observers record their observations directly. Thus, memory loss is counteracted. After the exercise has been processed, it will be assessed in terms of requirements or features. It has been shown that the more facts that have been recorded, the more nuanced and objective the assessment is. At a later discussion, it is possible that a consensus opinion be formed. Due to subjective sensations, groups are controlled and mitigated. To secure the quality of the observation, it is important to be aware of errors.

There are two groups of evaluation and judgement errors regarding observations. On the one hand, errors can be prevented or minimized by an adequate organisation of the observation. On the other hand, there are observation errors for which a consciousness can be created, but which cannot be stopped through measures.

\section{Results}

The assessment of the operational performance is difficult because of different experience-based protocols, which lack scientific verification. It is agreed that certain principles are effectual for a successful mission: For example to do the most you can for as many patients as possible. It is assumed that the used protocols and incidents plan are feasible. Based on this, the actual performance is compared to the theoretical performance to identify if the plans are fulfilled.

The documentation of the operational performance provides planners and rescue teams with a full picture of all processes. Through the evaluation, it is possible to identify strengths, weaknesses and bottlenecks. All of the results are summarized in an individual evaluation report.

The different tools are implemented in different exercises in the pre-hospital and hospital phase for mass casualty incident preparedness. Typical bottlenecks, which were identified in the hospital phase, are resources that cannot be increased as quickly as needed. Other problems are that the gateway for the ambulances is too crowded with incoming ambulances or there is not enough room to provide adequate care for the patients because of construction reasons. Additional personnel for the transport of a multitude of patients is needed. Different documentation forms, which lack standards, hinder data exchange between emergency medical services and hospitals.

\section{Conclusion}

The described Tele-Dialog system is suitable for the evaluation of emergency management exercises in small confined spaces. Moreover, the system is fast but 


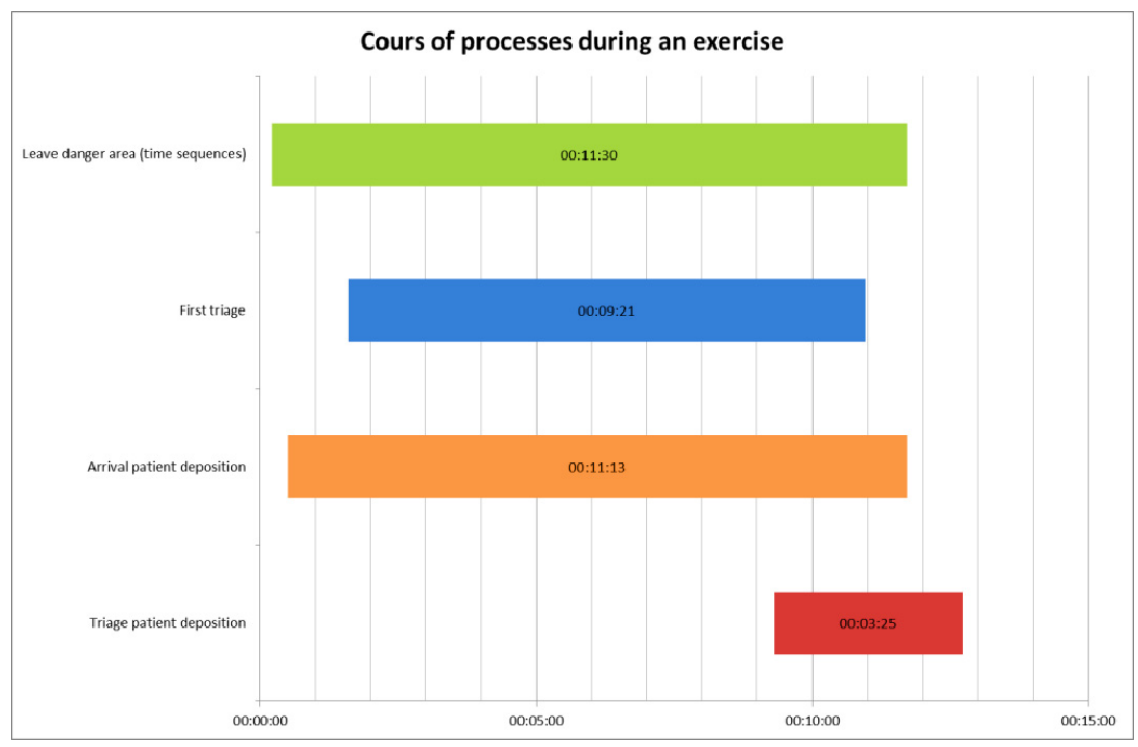

Figure 4: $\quad$ Course of processes during an exercise.

it has restrictions due to technical limitations, as it can only be used for certain previously programmed queries. Depending on the evaluation order however, it may be necessary to measure the three-dimensional positions of an object or a person, and also the time flow, in relation to each other, in order to gain operational insights at the micro-level. By means of such tracking technology, further conclusions on the plot of the forces can be drawn. It provides the adaptation of a local positioning technology, which is already being used in industrial manufacturing and logistics. A sensor network, which is required to operate such a system, can be created by using multiple sensors that measure the distance of tags to these sensor towers. These tags emit a radio signal, which is picked up by different sensor stations. Knowing the speed this signal travels, the position of the tag can be determined with an accuracy of $+/-30$ centimetres. The tags are small enough to be applied to persons as well as objects, allowing a three-dimensional tracking and documentation throughout the exercise.

This way, movement patterns of patients and rescue personnel can be tracked and evaluated to help to identify problems and to improve the rescuers' workflow.

To gain additional information, recognition algorithms are being developed and included into the program to automatically identify certain events, for instance, when a doctor is at the same position as a critically injured patient.

Furthermore, a recognition event for the meeting of two persons or objects can be programmed. An automatic analysis is possible and simplifies the subsequent evaluation of the action. 
A good evaluation requires different demands. The method should produce comparable and reproducible results for analysing large scale operations of emergency medical services [12]. Evaluation standards of DeGEval according to utility, feasibility, fairness, and accuracy have to be considered [13]. The methods used should be universally applicable and independent of operational concepts and rescue team procedures.

The evaluation method should make the target goal - to do as much as possible for as many as possible - measurable, while taking into account the care of the patients, which is representative for their welfare [12].

\section{References}

[1] Latasch, L.; Jung, G.; Ries, R.; Stark, S. (2006): Neuere medizinische Versorgungskonzepte (zur WM 2006) bei 1000 und mehr Verletzten. In: Notfall + Rettungsmedizin (9), S. 258-263.

[2] A. Beck, M. Bayeff-Filloff, K.-G. Kanz, and S. Sauerland, "Algorithmus für den Massenanfall von Verletzten an der Unfallstelle," Notfall + Rettungsmedizin, vol. 8, no. 7, pp. 466-473, Nov. 2005.

[3] K.-G. Kanz, P. Hornburger, M. V. Kay, W. Mutschler, and W. Schäuble, "mSTaRT-Algorithmus für Sichtung, Behandlung und Transport bei einem Massenanfall von Verletzten," Notfall + Rettungsmedizin, vol. 9, no. 3, pp. 264-270, May 2006.

[4] S. Wolf, A. Partenheimer, C. Voigt, R. Kunze, H. A. Adams, and H. Lill, "Die Erstversorgungsklinik bei einem Großschadensereignis MANV IV," Der Unfallchirurg, vol. 112, pp. 565-574, 2009.

[5] H. A. Adams, A. Flemming, C. Lange, F. Hildebrand, C. Krettek, and W. Koppert, "Das EVK-Konzept - ein Beitrag zur Patientenversorgung im Großschadens- und Katastrophenfall," Anästhesiologie \& Intensivmedizin, vol. 53, no. 1, pp. 4-18, 2012.

[6] Arbeitsgemeinschaft der Leiter der Berufsfeuerwehren in der Bundesrepublik Deutschland, "Führung und Leitung im Katastrophenschutz in der Bundesrepublik Deutschland," no. 0251, p. 20, 2005.

[7] Forschungszentrum Informatik, "www.manet-projekt.de," 2010. [Online]. Available: http://www.manet-projekt.de/. [Accessed: 30-May-2012].

[8] K. Maurer, "Organisatorischer Leiter Rettungsdienst (OrgL) - unverzichtbar neben dem LNA?," Journal für Anästhesie und Intensivbehandlung, vol. 9, no. 4, pp. 43-46, 2001.

[9] P. Hügler, "MANV-Konzepte," Journal für Anästhesie und Intensivbehandlung, vol. 9, no. 4, p. 42, 2001.

[10] Hirshberg, A.; Mattox, K. (2009): Modeling and Simulation in Terror Medicine. In: Essentials of terror medicine, S. 79-94.

[11] Lechleuthner, Alex; Weber, B.; Käser, B. (2010): LÜKEX 2010. Beobachtungen und Auswertungen. Übungsbericht. Fachhochschule Köln. Institut für Rettungsingenieurwesen und Gefahrenabwehr. 
[12] Stiehl, Manuel (2012): Anforderungen an eine Evaluationsmethode für rettungsdienstliche Großeinsätze. Fachhochschule Köln. Institut für Rettungsingenieurwesen und Gefahrenabwehr.

[13] DeGEval - Gesellschaft für Evaluation e.V.: Standards für Evaluation [Internet]. 4. unverän Mainz, DeGEval - Gesellschaft für Evaluation, 2008. 